\title{
Radio-Acoustic-Sounding System - berührungslose und abstandsaufgelöste akustische Temperaturmessung im industriellen Umfeld
}

\author{
D. Exel, S. Schuster, K. Thaller, B. Zagar, D. Zankl, S. Scheiblhofer, V. Ganglberger, J. Reisinger
}

\begin{abstract}
Dieser Artikel beschreibt einen Messaufbau für die berührungslose, abstandsaufgelöste Temperaturmessung von Gasen. Die elektromagnetisch-akustische Wechselwirkung, bekannt von der Radio-Acoustic-Sounding Methode, macht es möglich, auch im industriellen Umfeld kontinuierlich Temperaturen zu messen. Dazu wird die Schallgeschwindigkeit, welche in einem eindeutigen Zusammenhang mit der Temperatur steht, mit einem Doppler-Radar bestimmt. Das Funktionsprinzip, ein realisierter Aufbau und dessen Herausforderungen werden aufgezeigt und mit Messungen dargestellt. Weiteres wird diskutiert, wie mit diesem Messprinzip neben der Temperatur auch Luftströmungen mehrdimensional bestimmt werden können.
\end{abstract}

Schlüsselwörter: berührungslose akustische Temperaturmessung; Doppler-Radar; RASS

Radio-acoustic-sounding system—spatially resolved acoustic temperature measurement for industrial applications.

This article describes a measurement setup for non-contact, spatially resolved temperature measurement of gases. The electromagnetic-acoustic interaction, known from the radio-acoustic-sounding method, makes it possible to measure temperatures continuously in the industrial environment. For this purpose, the speed of sound of an emitted sound pulse, which is temperature-dependent, will be determined with a Doppler radar. The operating principle, a possible setup and its challenges are shown and presented with measurements. Further, it is discussed how air flows or temperatures can be determined multidimensionally with this measuring principle.

Keywords: non-contact acoustic temperature; measurement; Doppler radar; electromagnetic-acoustic interaction

\section{Einleitung}

Einsatz in einer rauchigen, staubigen Umgebungsluft, sowie Zuverlässigkeit und kleine Unsicherheit sind nur einige Aspekte, die für die Temperaturmessung im industriellen Umfeld wichtig sind. Während einige unterschiedliche Verfahren zur berührungslosen Temperaturmessung von Oberflächen bekannt sind $[1,2]$ und deren Messunsicherheit bereits detailliert untersucht wurden $[3,4]$, nutzen berührungslose Temperaturmessmethoden in Gasen meist die Eigenschaft, dass die Schallgeschwindigkeit $c_{a}$ von der Temperatur eindeutig abhängig ist [5]

$$
c_{a}=\sqrt{\frac{R \kappa \vartheta}{M}} \approx 20,055 \sqrt{\vartheta}
$$

$R$ ist dabei die universelle Gaskonstante $\left(8,3145 \frac{\mathrm{J}}{\mathrm{molK}}\right), M$ die molare Masse $\left(0,02896 \frac{\mathrm{kg}}{\mathrm{mol}}\right.$ bei Luft) und $\kappa$ der Adiabatenexponenten $\left(1,402\right.$ für trockene Luft). Die Bestimmung von $c_{a}$ erfolgt entweder indirekt (bei homogener Temperaturverteilung) über die Messung der Laufzeit eines Schallpulses oder ortsaufgelöst über die elektromagnetisch-akustischen Wechselwirkung [6]. Das Messprinzip hinter dieser Wechselwirkung ist aus der Meteorologie bekannt und wird auch als RASS (Radio Acoustic Sounding System) bezeichnet $[7,8] .1980$ wurde damit erstmals die Temperatur in der unteren Troposphäre gemessen $[9,10]$. Seit 1990 findet das RASS weitverbreitet Einsatz an Flughäfen.

\section{Funktionsprinzip}

Das Funktionsprinzip des RASS beruht auf der Geschwindigkeitsmessung eines Schallpulses mittels eines Doppler-Radarsystems. Abbildung 1 zeigt eine Prinzipskizze der vorgestellten Methode. Durch den vom Lautsprecher ausgesendeten Schallpuls (rot dargestellt) wird der Brechungsindex der Luft periodisch moduliert, was die Streuung von elektromagnetischen Wellen (schwarz dargestellt) ermöglicht. Damit eine möglichst hohe elektromagnetische Leistung zurück gestreut werden kann, muss die Radarwellenlänge $\lambda_{\mathrm{e}}$ mit

Exel, Dominik, Institut für Elektrische Messtechnik, Johannes Kepler Universität Linz, Altenbergerstraße 69, 4040 Linz, Austria (E-Mail: dominik.exel@jku.at); Schuster, Stefan, voestalpine Stahl GmbH, voestalpine-Straße 3, 4031 Linz, Austria (E-Mail: stefan.schuster2@voestalpine.com); Thaller, Kurt, Institut für Elektrische Messtechnik, Johannes Kepler Universität Linz, Altenbergerstraße 69, 4040 Linz, Austria (E-Mail: kurtthaller@hotmail.com); Zagar, Bernhard, Institut für Elektrische Messtechnik, Johannes Kepler Universität Linz, Altenbergerstraße 69, 4040 Linz, Austria

(E-Mail: bernhard.zagar@jku.at); Zankl, Dominik, voestalpine Stahl GmbH, voestalpine-Straße 3, 4031 Linz, Austria (E-Mail: dominik.zankl@voestalpine.com); Scheiblhofer, Stefan, voestalpine Stahl GmbH, voestalpine-Straße 3, 4031 Linz, Austria (E-Mail: stefan.scheiblhofer@voestalpine.com); Ganglberger, Vera, voestalpine Stahl GmbH, voestalpine-Straße 3, 4031 Linz, Austria

(E-Mail: vera.ganglberger@voestalpine.com); Reisinger, Johann, voestalpine Stahl $\mathrm{GmbH}$, voestalpine-Straße 3, $4031 \mathrm{Linz}$, Austria

(E-Mail: johann.reisinger@voestalpine.com) 

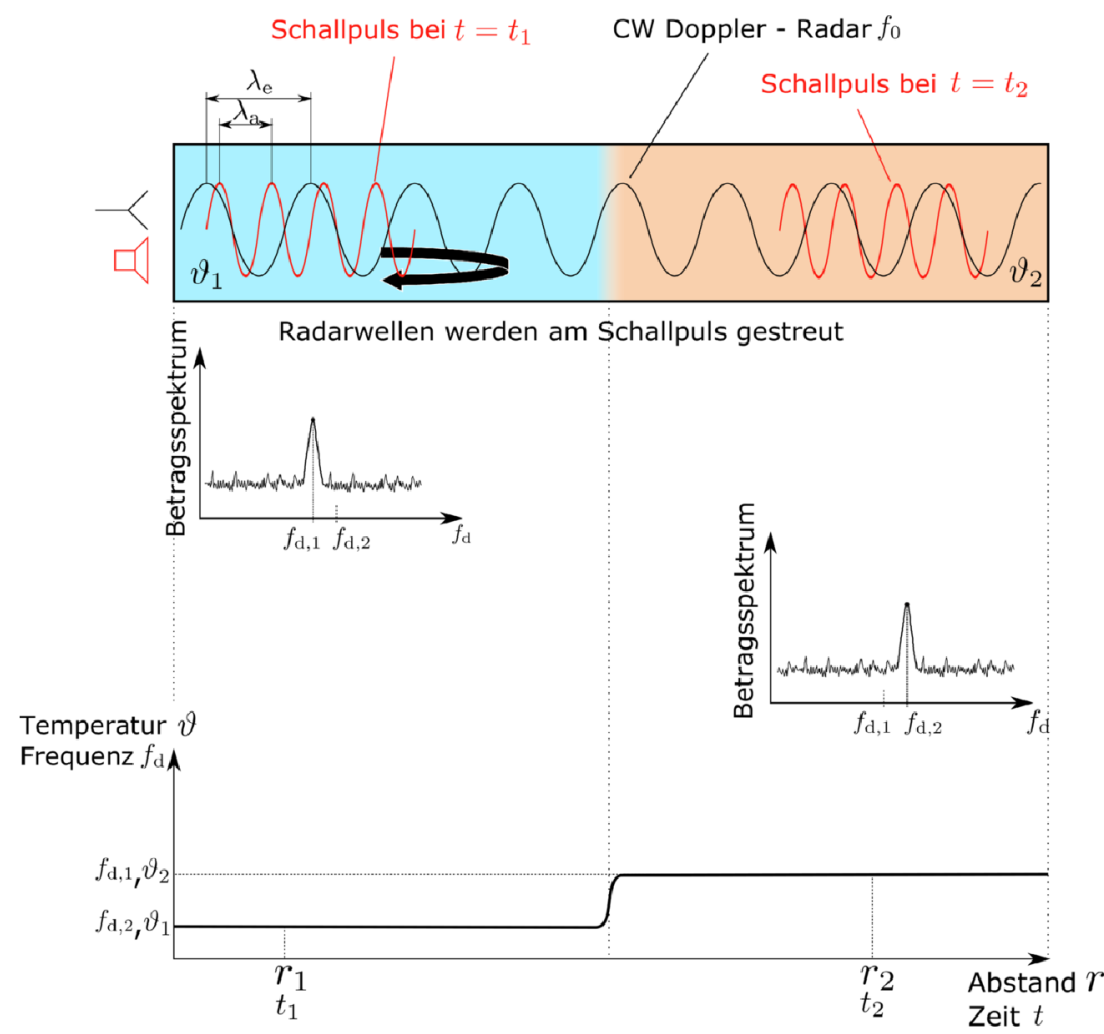

Abb. 1. Prinzipskizze RASS: Die Geschwindigkeit der ausgesendeten Schallpulse wird mittels eines Doppler-Radar gemessen. Die so erhaltenen Dopplerfrequenzen $f_{d, 1}, f_{d, 2}$ stehen in einem eindeutigen Zusammenhang mit der Temperatur (Farbig online)

der akustischen Wellenlänge $\lambda_{a}$ Bragg abgestimmt sein

$$
\lambda_{\mathrm{e}}=2 \cdot \lambda_{\mathrm{a}}
$$

Es wurden Wellenlängen von $\lambda_{e}=30 \mathrm{~mm}$ und $\lambda_{a}=15 \mathrm{~mm}$ gewählt, und werden unter Abschn. 3 genauer diskutiert. Ist die Bragg Bedingung (2) erfüllt, kommt es zu einer konstruktiven Interferenz der an den Phasenflächen der Schallwelle entgegen der Ausbreitungsrichtung zurückgestreuten elektromagnetischen Wellen [11,

12]. Grundsätzlich gibt es zwei Betriebsarten: Continuous wave (CW) Radar mit gepulstem Schall (Abb. 1) oder CW Schall mit gepulstem Radar [7]. Erstere hat den Vorteil, dass alle früher ausgesendeten Schallpulse genügend Zeit haben abzuklingen und es somit zu keinen nennenswerten störenden Reflexionen selbst in geschlossenen Räumen kommt. Die Relativbewegung zwischen dem stationären Doppler-Radar und dem ausgesendeten Schallpuls, welcher als bewegter Reflektor für das Radar dient, führt zu einer DopplerFrequenzverschiebung $f_{\mathrm{d}}$ des empfangenen Radarsignals $[13,14]$. Die Frequenz des empfangenen Signals ist gegeben mit

$$
f_{\mathrm{d}}=\frac{2 c_{\mathrm{a}}}{c_{\mathrm{e}}} f_{\mathrm{e}} .
$$

$f_{\mathrm{e}}$ beschreibt dabei die Sendefrequenz des CW Doppler-Radars und $c_{\mathrm{e}}$ die Ausbreitungsgeschwindigkeit des Lichtes (299792458 $\frac{\mathrm{m}}{\mathrm{s}}$ ). Gleichungen (1) und (3) kombiniert beschreiben also den Zusammenhang zwischen Dopplerfrequenz und Temperatur. Die an der Empfangsantenne des Radars zurückgestreute Leistung berechnet sich mit

$$
P_{r}=\frac{P_{t} G_{t} G_{r} \lambda_{e}^{2}}{(4 \pi)^{3} r^{4}} \sigma,
$$

wobei $P_{\mathrm{t}}$ die gesendete Leistung, $G_{\mathrm{t}}$ bzw. $G_{\mathrm{r}}$ den Gewinn der Sendebzw. Empfangsantenne, $r$ den aktuellen Abstand des Schallpulses zur Radarantenne und $\sigma$ den Radarquerschnitt der Schallwellen bezeichnet [15]. Marshall [16] und Brooker [17] leiten in ihrer Arbeit einen theoretischen Ausdruck für den Radarquerschnitt einer akustischen Wellenfront

$$
\sigma=A N_{\text {Bragg }}^{2} r^{2} P_{a} \frac{\sin \left[\left(2 k_{\mathrm{e}}-k_{\mathrm{a}}\right) N_{\text {Bragg }} \lambda_{\mathrm{a}} / 2\right]}{\left(2 k_{\mathrm{e}}-k_{\mathrm{a}}\right) N_{\text {Bragg }} \lambda_{\mathrm{a}} / 2}\left(1-\cos \left(\frac{\theta}{2}\right)\right)^{2}
$$

her. $P_{a}$ bezeichnet die akustische Sendeleistung, $A$ eine Konstante für verschiedene atmosphärische Parameter und auch die Gladstone-Dale Beziehung [18], $N_{\text {Bragg }}$ die Anzahl der Schallperioden und $k_{\mathrm{e}}=2 \pi / \lambda_{\mathrm{e}}$ bzw. $k_{\mathrm{a}}=2 \pi / \lambda_{\mathrm{a}}$ die Wellenzahlen. Die örtliche Länge des Schallpulses ist somit $N_{\text {Bragg }} \cdot \lambda_{\mathrm{a}}$. Setzt man (5) in (4) ein und kürzt $r^{2}$, so erhält man die RASS-Gleichung. Eine wesentliche Eigenschaft der RASS-Gleichung im Vergleich zur RadarGleichung [19] ist, dass die empfangene Leistung $\propto \frac{1}{R^{2}}$ und nicht $\propto \frac{1}{R^{4}}$ ist. Die $\propto \frac{1}{R^{2}}$ Abhängigkeit ist auf die sphärisch divergierende akustische Welle zurückzuführen. Eine wichtige Voraussetzung für die Gültigkeit diese Gleichung ist, dass sich die akustische und die Radar-Hauptkeulen ausreichend überlappen, wobei der kleinere Raumwinkel $\theta$ entscheidet und in (5) verwendet werden muss. Eine weitere Anforderung ist, dass die elektromagnetische mit der akustischen Welle ideal kollimiert und koaxial bzw. nur gering divergent überlappend sind. Ein möglicher Aufbau wird in Abschn. 3 und [13] diskutiert. Des Weiteren ist die empfangene Leistung $\propto N_{\text {Bragg }}^{2}$, d.h. $P_{r}$ ist stark von der Anzahl der akustischen Perioden abhängig. Abbildung 2 zeigt, dass bei Verdopplung der Bragg-Zyklen die empfangene Leistung um $6 \mathrm{~dB}$ zunimmt, wenn die Bragg-Bedingung (2) mit Gleichheit erfüllt wird. Das Einschwingverhalten des Lautsprechers 

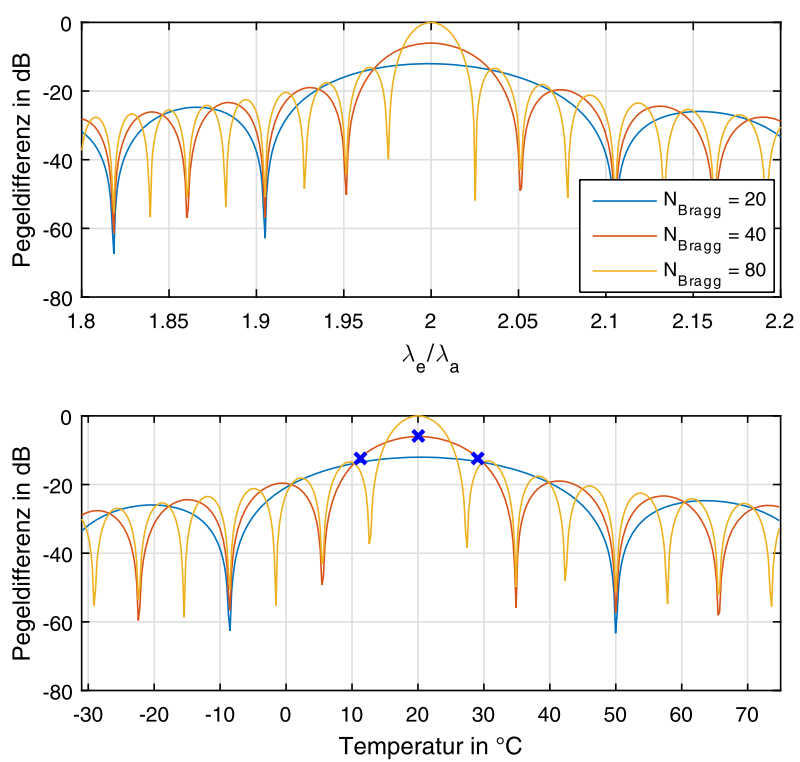

Abb. 2. (Oben) Wird die Bragg-Bedingung $\lambda_{e}=2 \cdot \lambda_{a}$ nicht genau getroffen, so sinkt die Empfangsleistung abhängig von der Anzahl der Schallzyklen. (Unten) Mit einer kürzeren Schallpulslänge (weniger Bragg-Zyklen) kann ein größerer Temperaturbereich abgedeckt werden, allerdings sinkt die zurückgestreute Leistung (Farbig online)

führt zu einer, hier mit Fensterfunktion bezeichneten, Modulation des Schallpulses, die sich auch bei der Spektralanalyse auswirkt, hier aber der Einfachheit halber vernachlässigt wird. Es würde zu einer Verbreiterung, sowie gleichzeitiger Reduktion der Hauptkeule in Abb. 2 führen. Aufgrund der unbekannten Temperaturinhomogenitäten in der Atmosphäre, wie z.B. Wind, ist es allerdings schwer die Bragg-Bedingung exakt zu treffen. Ein Nachteil dieser RASS Messmethode ist, dass ein grobes Vorwissen der ungefähren Temperatur vorhanden sein sollte. Bei 60 Schallzyklen (roter Graph) wird mit einer Schwelle von $-6 \mathrm{~dB}$ (blaue Kreuze) gegenüber dem erzielbaren Optimum ein Temperaturbereich von $11^{\circ} \mathrm{C}$ bis $29^{\circ} \mathrm{C}$ abgedeckt. Der Schwellwert sollte so gewählt werden, dass ein ausreichender Signal-Rausch-Abstand (SRA) erzielt wird. Ist die ungefähre Temperatur des Gases nicht bekannt, so müssen Schallpakete unterschiedlicher Frequenzen ausgesendet werden, um einen größeren Temperaturbereich abdecken zu können.

\section{Aufbau}

Das Doppler-Radar wurde als monostatisches Radar realisiert, d.h die Sendeantenne ist gleichzeitig auch die Empfangsantenne. Abbildung 3 zeigt den mechanischen Messaufbau. Das System und die Einstellungen (eingestellte Schallpulslänge, Anzahl gemittelter Messungen, usw. wird unter Abschn. 4 genauer beschrieben) wurden so konzipiert, dass Temperaturen zwischen $-10^{\circ} \mathrm{C}$ und $50^{\circ} \mathrm{C}$ gemessen werden können. Durch Änderung der Frequenzen (sowohl Schall wie auch Radar) können auch andere Temperaturbereiche abgedeckt werden. Ausgehend von einem $10 \mathrm{GHz}$ Synthesizer $\left(\lambda_{\mathrm{e}}=30 \mathrm{~mm}\right)$ für die Erzeugung des Radarsignals, sind aufgrund der Bragg-Bedingung (2) Schallfrequenzen zwischen $21,7 \mathrm{kHz}$ und $24 \mathrm{kHz}$ notwendig $\left(\lambda_{a}=15 \mathrm{~mm}\right)$. Als Schallquelle wird der Hochtöner GZCT 0500X verwendet. Da nur kurze, intensive Schallpulse ausgesendet werden, wird auf das Einschwingverhalten des Hochtöners durch Fensterung im Zeitbereich (z.B. Hamming [20]) Rücksicht genommen

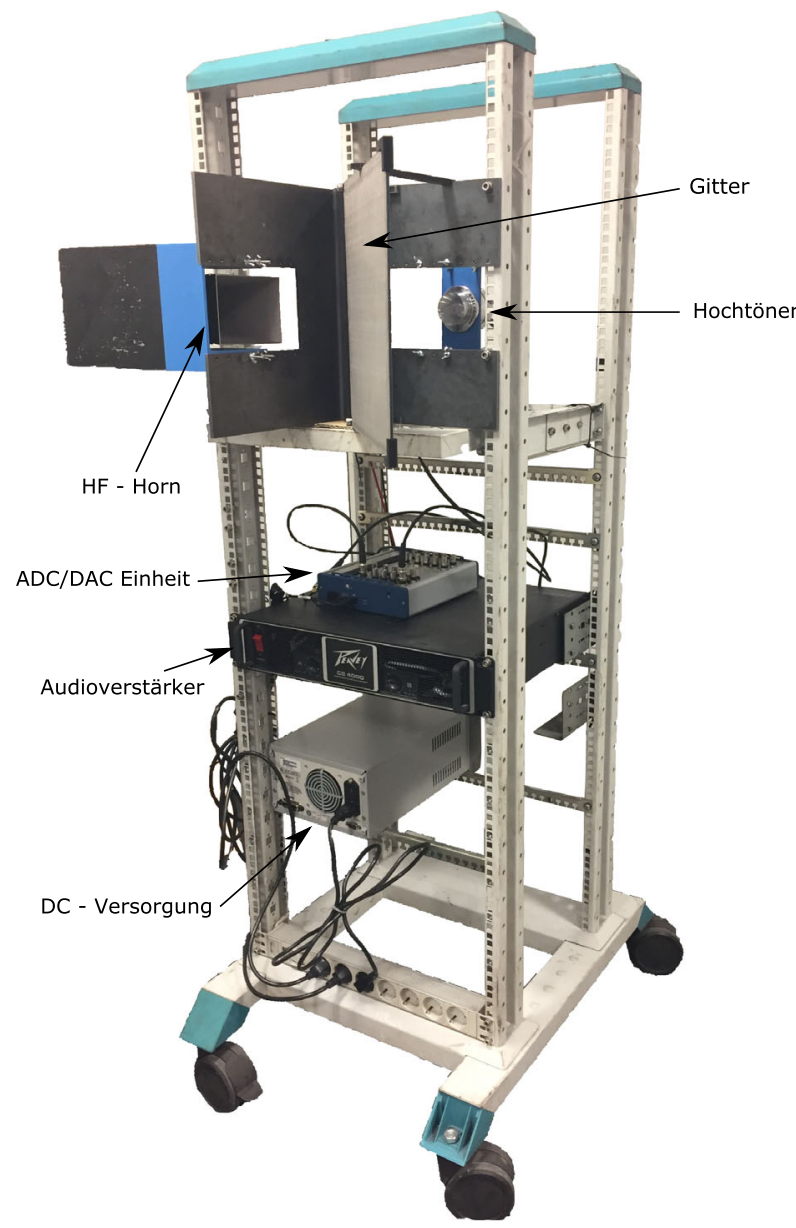

Abb. 3. Messaufbau des Radio Acoustic Sounding Systems. Das Gitter bewirkt eine optimale Kollokation der elektromagnetischen Wellen mit den Schallwellen [17]. Somit ist die Position beider Quellen zumindest näherungsweise virtuell gleich

Abbildung 4 zeigt ein Blockschaltbild des Doppler-Radars mit den jeweiligen Leistungspegeln der verwendeten Komponenten. Als HF Quelle dient der Mikrowellen Synthesizer FSL-0020 der Firma National Instruments mit einer Ausgangsleistung von $10 \mathrm{~dB}$ m. Die Gesamtverluste im Sendepfad betragen $\approx 8 \mathrm{~dB}$ und setzen sich zusammen aus den Kabelverlusten, der Einfügedämpfung des Zirkulators und den Verlusten des HF-Splitters. Des Weiteren befindet sich ein 23 dB Verstärker im Sendepfad. Das System wurde so ausgelegt, dass die höchstmögliche Radarleistung verwendet wird. Die Antenne besitzt einen Antennengewinn von $20 \mathrm{dBi}$ ( $\mathrm{i}$... isentrop) bei einem $-3 \mathrm{~dB}$ Öffnungswinkel von $14^{\circ}$. Somit beträgt die äquivalente isotrope Strahlungsleistung (EIRP) $43 \mathrm{~dB} \mathrm{~m}$. Bei einem Radarquerschnitt von $-60 \mathrm{~dB} \mathrm{~m}^{2}$ beträgt die Empfangsleistung, welche mit der RASS-Gleichung berechnet werden kann, am Antennenfußpunkt $-87 \mathrm{~dB}$ m. Dabei wird von $N_{\text {BRAGG }}=100$ Schallperioden in einem Abstand von $r=5 \mathrm{~m}$ ausgegangen. Im Empfangspfad fallen nochmals $1 \mathrm{~dB}$ Kabelverluste und $7 \mathrm{~dB}$ Mischerverluste an. Nach einem 20 dB Verstärker (50 $\Omega$ Ausgang) wird das Doppler-Signal mit einem 16 Bit Analog zu Digital Umsetzer bei einer Referenzspannung von $1 \mathrm{~V}$ abgetastet.

\subsection{Kollokation}

Wie in Abb. 3 dargestellt, wird ein feines Metallgitter verwendet, welches für elektromagnetische Wellen wie ein Spiegel und für 


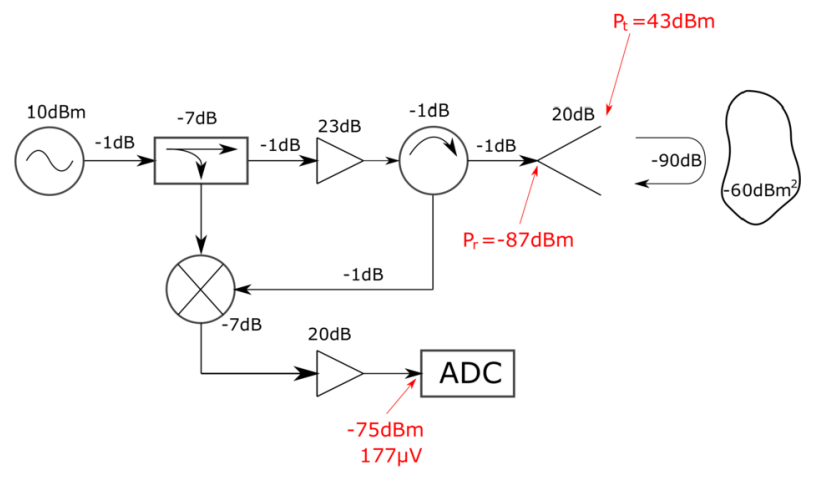

Abb. 4. Pegelplan für die Anwendung des Heterodynprinzips. Bei einer Schallpulslänge von $1,5 \mathrm{~m}$ in einem Abstand von $5 \mathrm{~m}$ ergibt sich eine RCS von $-60 \mathrm{~dB} \mathrm{~m}^{2}$, sowie ein Pfadverlust von $-90 \mathrm{~dB}$. Somit beträgt die Empfangsleistung mit diesem Setup $-75 \mathrm{~dB} m$

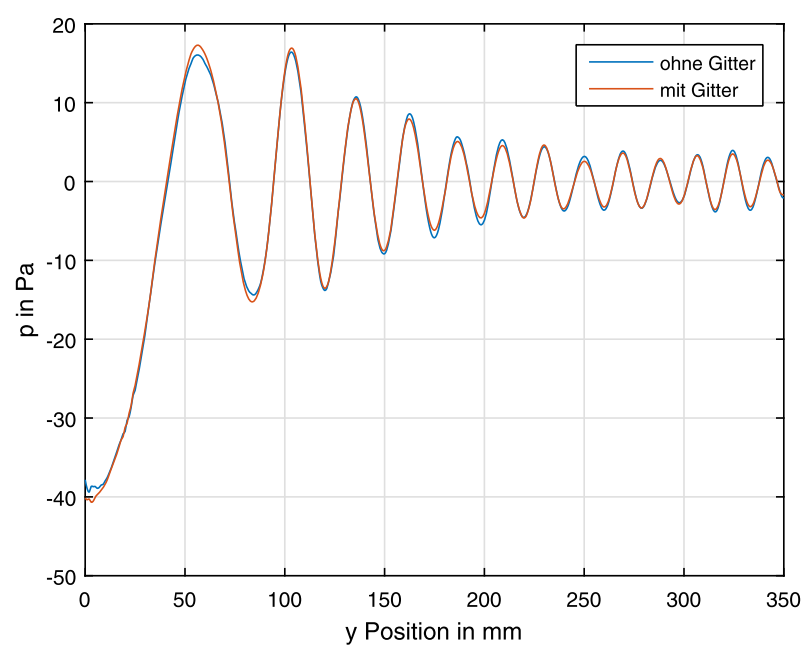

Abb. 5. Messergebnis zum Vergleich des Schallfeldes mit und ohne Gitter. Das Gitter bewirkt keine nennenswerte Dämpfung des Schallfeldes und stellt somit keinen Diffusor dar (Farbig online)

akustische Wellen wie nicht vorhanden wirkt. Dadurch ist es möglich eine Kollokation der sphärischen Kugeloberflächen der akustischen bzw. Radarwellen zu ermöglichen. Das hier verwendete Gitter besitzt eine Drahtstärke von 0,2 $\mathrm{mm}$ und hat eine Gitterweite von $0,75 \mathrm{~mm}$

Um die Schallausbreitung durch das Gitter zu untersuchen, wurden im Rahmen einer wissenschaftlichen Arbeit [18] die Schalldrücke mit und ohne Gitter an verschiedenen Positionen gemessen. Die Messdaten wurden mit einem Mikrofon, welches in einem Abstand $z_{0}=250 \mathrm{~mm}$ in Ausbreitungsrichtung vom Lautsprecher entfernt war, aufgenommen. Für die Schallfeldmessung mit Gitter wurde dieses zwischen Lautsprecher und Mikrofon im Abstand von $z_{1}=100 \mathrm{~mm}$ zur Lautsprechermembran montiert. Der Hochtöner wurde mittels Linearachsen in horizontaler Richtung y zwischen 0 und $350 \mathrm{~mm}$ verfahren. Abbildung 5 zeigt einen Vergleich der Schalldruckamplituden $p$ des Schallfeldes bei impulsförmiger Anregung für einen festen Zeitpunkt. Es ist kein nennenswerter Unterschied erkennbar. Der geringe Einfluss des Gitters auf das Schallfeld lässt sich dadurch begründen, dass die Wellenlänge des ausgesendeten Schallpulses ( $\lambda_{a}=15 \mathrm{~mm}$ ) um ein Vielfaches größer ist als die Drahtstärke und somit nur ein sehr geringer Teil reflektiert wird.

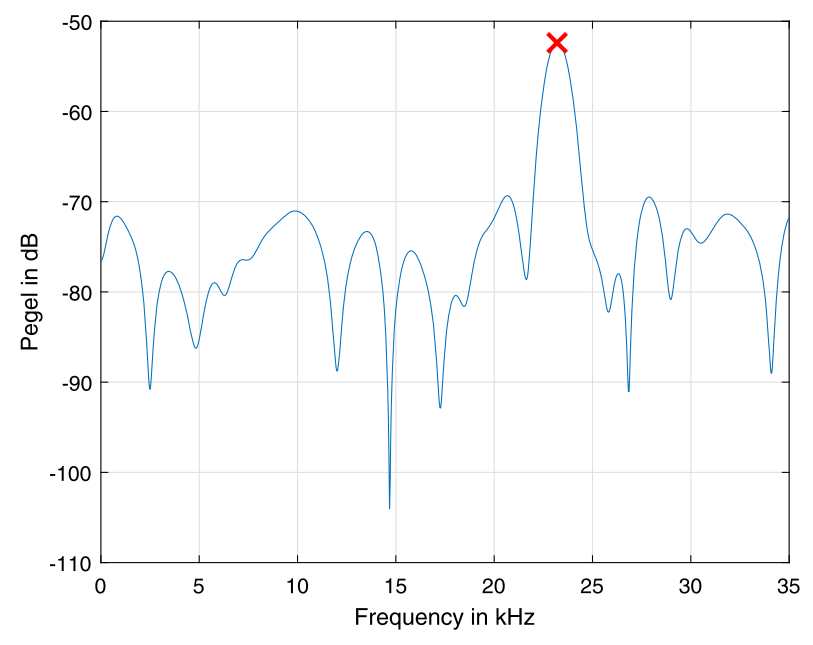

Abb. 6. Typisches Leistungsdichtespektrum nach der Spektralanalyse. Ein ausgeprägter Peak ist bei $23,22 \mathrm{kHz}$ erkennbar. Diese Dopplerfrequenz ist proportional zu der Temperatur des Gases indem die Geschwindigkeit des Schallpulses gemessen wird. Parameter: Schallpulslänge $=0,4 \mathrm{~m}$, Anzahl gemittelter Messungen $=800$

\section{Temperaturprofilmessung}

Es wurden Messungen in einem abgeschlossenen Laborraum durchgeführt, d.h. Fenster und Türen waren geschlossen, um mögliche Luftverwirbelungen zu vermeiden. Um Rauschen zu reduzieren, wurden 800 Messungen gemittelt. Alle 20 ms wurde ein Schallpaket ausgesendet, somit beträgt die Messzeit für 800 Messungen $16 \mathrm{~s}$. Innerhalb der kurzen Messzeit kann die Raumtemperatur als konstant angenommen werden. Bereits unter Abschn. 2 wurde erwähnt, dass das Abklingen der Schallintensität notwendig ist, um Mehrfachreflexionen zu vermeiden.

Abbildung 6 zeigt ein typisches Leistungsdichtespektrum des ausgewerteten Doppler-Signals. Bei 23,22 kHz ist ein ausgeprägter Peak zu erkennen, welcher der Dopplerfrequenz des Schallpulses entspricht. Da die ausgesendete Frequenz des Doppler-Radars $f_{\mathrm{e}}$ und die Lichtgeschwindigkeit $c_{\mathrm{e}}$ ausreichend genau bekannt sind, kann mit Gleichung (3) die Schallgeschwindigkeit $c_{a}$ berechnet werden. Mit Gleichung (1) kann anschließend die Temperatur $\left(\vartheta=28,35^{\circ} \mathrm{C}\right)$ bestimmt werden.

Es wurden mehrere Messreihen hintereinander aufgenommen und ausgewertet. Abbildung 7 zeigt die zeitliche Entwicklung der Temperatur entlang der Tiefendimension (von 0,5 $\mathrm{m}$ bis $3 \mathrm{~m}$ ) für einen Zeitraum von $310 \mathrm{~s}$. Bei einer Messzeit von $16 \mathrm{~s}$ für 800 gemittelte Messungen konnten somit 19 Messreihen aufgenommen werden. Verifiziert wurde die Messung mit einem handelsüblichen Thermometer. Die örtliche Position der Schallpulse wurde über deren Laufzeit bestimmt. Dabei wurde eine konstante Raumtemperatur von $\vartheta=28^{\circ} \mathrm{C}$ angenommen. Bei Messungen für größere Entfernungen kommt es mit diesem Ansatz (Annahme konstante Temperatur im Raum) zu nennenswerten Abweichungen zwischen berechneter und tatsächlicher Laufzeit des Schallpulses. Speziell bei inhomogener Temperatur des Gases treten erhebliche Ungenauigkeiten auf. Deshalb sollte in diesen Fällen die Laufzeit mit $T(r)=\int_{0}^{r} \frac{1}{c_{\mathrm{a}}(s)} \mathrm{d} s$ berechnet werden.

Bei $60 \mathrm{~s}$ und in einem Abstand von 2,2 m sieht man einen Messausreißer. Theoretisch wurden hier $-25^{\circ} \mathrm{C}$ berechnet. Das bedeutet, es wurde der höchste Frequenzpeak bei $21,07 \mathrm{kHz}$ detektiert. Das ist auf einen zu geringen SRA zurückzuführen, da diese Temperatur definitiv ausgeschlossen werden kann. Der sogenannte threshold - 


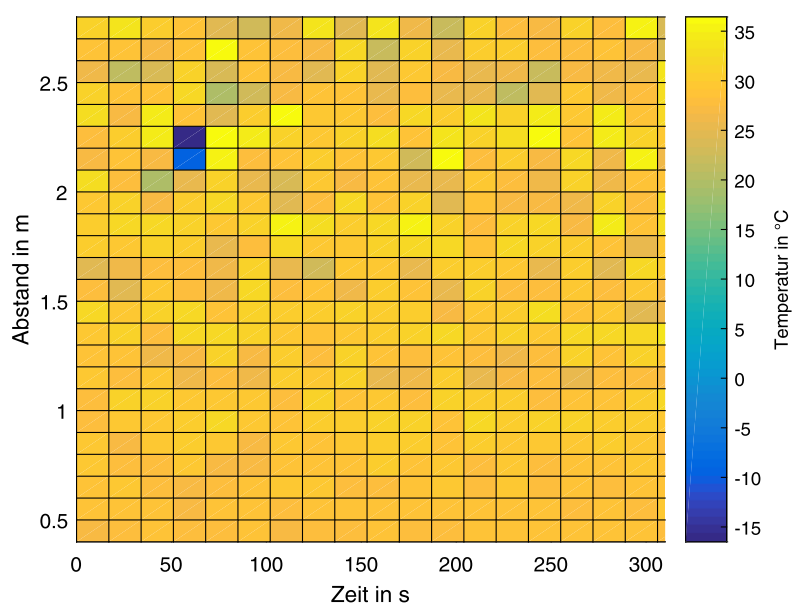

Abb. 7. Zeitliche Temperaturprofilmessung im Raum. Erkennbar ist, dass ab ca. $2 \mathrm{~m}$ Abstand die Messung unzuverlässig wird. Die gemittelte Temperatur im Raum beträgt $\vartheta=28,45^{\circ} \mathrm{C}$. Parameter: Schallpulslänge $=0,4 \mathrm{~m}$, Anzahl gemittelter Messungen $=800$ (Farbig online)

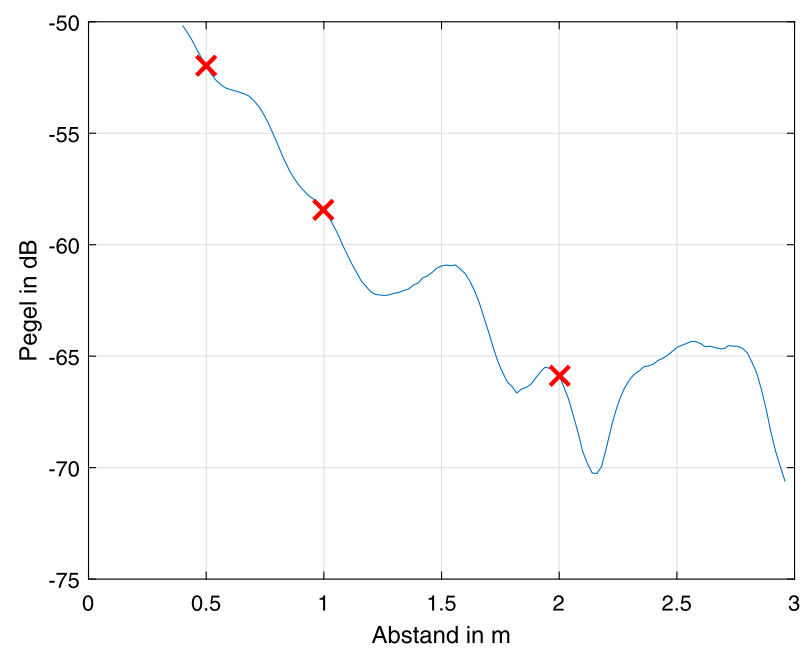

Abb. 8. Pegel der Peaks der Spektralanalyse als Funktion des Abstandes $r$. Eine Verdopplung des Abstandes bewirkt eine Abnahme um $6 \mathrm{~dB}$. Parameter: Schallpulslänge $=0,4 \mathrm{~m}$, Anzahl gemittelter Messungen $=800$

Level für die Spektralanalyse wurde hier unterschritten [21, 22]. Das Messsystem mit diesen Einstellungen (Bragg-Zyklen, Anzahl der Mittelungen, ... ) ist in der Lage bis zu 2 m Entfernung die Temperatur zuverlässig zu messen. Für größere Distanzen ist der SRA-Abstand nicht mehr ausreichend, um einen signifikanten Peak mit hoher Sicherheit zu detektieren und somit die Temperatur verlässlich zu berechnen.

Abbildung 8 zeigt für den Zeitpunkt $t=150 \mathrm{~s}$ die Pegel der Peaks des Spektrums für den jeweiligen Abstand $r$. Eine Verdopplung des Abstandes bewirkt eine Abnahme des Pegels um $6 \mathrm{~dB}$. Wie schon erwähnt, sind mit diesen Einstellungen und Setup, Messungen bis 2 m sinnvoll.

\section{Ausblick}

Mit dem vorgestellten RASS System ist es möglich eindimensional die Schallgeschwindigkeit zu messen und anschließend die Tempera-

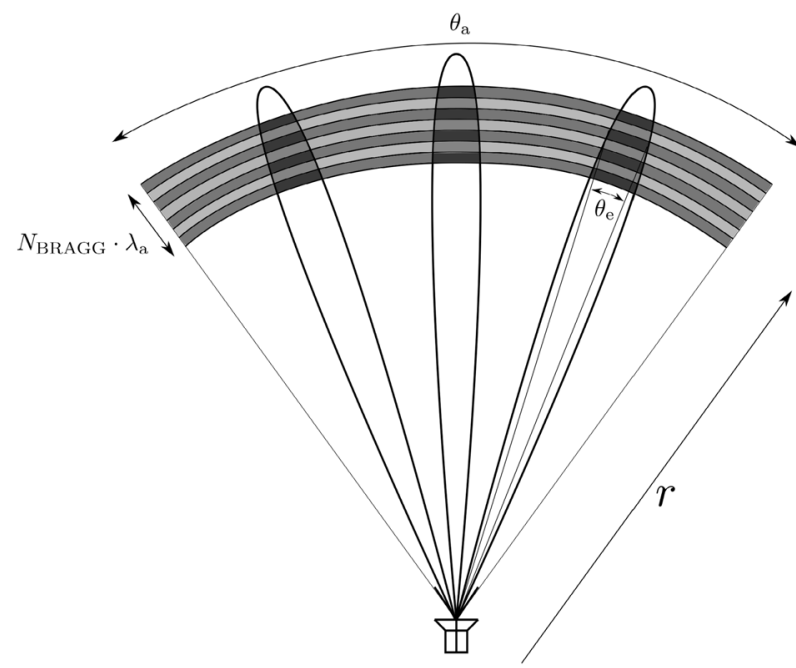

Abb. 9. Mit dem bekannten Beamforming aus der Radartechnik ist es möglich auch mehrdimensional Temperatur zu messen. Dabei wird ein breiter Abstrahlwinkel eines Hochtöners genutzt

tur zu berechnen. Um flächig die Temperatur zu bestimmen, könnte die bekannte Beamforming-Technik angewendet werden. Mit diesem Verfahren aus der Radartechnik ist es möglich, die Hauptkeule der Radarstrahlen zu schwenken [21]. Auch ein mechanisches Schwenken des RASS wäre denkbar [7]. In Abb. 9 ist eine Prinzipskizze dargestellt, die einen Schallpuls, mit der Länge $N_{\text {Bragg }} \cdot \lambda_{a}$ in einem Abstand $r$, zeigt. Dabei kann ein großer Öffnungswinkel des Lautsprechers $\theta_{a}$ ausgenutzt werden, um einen ausgedehnten Bereich abdecken zu können. Gleichzeitig sollte der Öffnungswinkel der Radarantenne $\theta_{\mathrm{e}}$ signifikant kleiner als der des Lautsprechers sein, damit die Winkelauflösung erhöht werden kann. Wie bereits im Abschn. 4 genauer diskutiert, kann unter Berücksichtigung der Laufzeit des Schallpulses das Messfenster in radialer Richtung variiert werden.

Für industrielle Applikationen ist es wichtig, die Messunsicherheit eines Messsystems zu kennen. Da es sich bei dieser Temperaturmessung um ein Frequenzschätzproblem handelt, kann mittels der Cramer-Rao lower Bound [21-23] die untere Schranke der Varianz der Temperatur berechnet werden. Diese Genauigkeitsgrenzen, erreichbare Auflösung sowie Methoden der Signalverarbeitung werden in einer zukünftigen Arbeit diskutiert.

\section{Zusammenfassung}

Das vorgestellte Messprinzip ist aus der Meteorologie bekannt und erlaubt berührungslos die Temperatur von Gasen zu bestimmen. Es hat auch Potential für den industriellen Einsatz. Mit diesem realisierten Setup und Einstellungen, ist es möglich Temperaturen bis zu einem Abstand von $2 \mathrm{~m}$ zuverlässig zu messen. Mit einer höheren Schallleistung bzw. Radarleistung kann der SRA erhöht und somit auch der maximale Abstand für zuverlässige Temperaturmessungen vergrößert werden. Es wurde gezeigt, dass mit einer größeren Anzahl der Schallzyklen auf der einen Seite die zurückgestreute Radarleistung vergrößert werden kann, auf der anderen Seite allerdings die Bragg-Bedingung genauer getroffen werden muss.

Des Weiteren wurde gezeigt, dass ein Metallgitter, welches für die Kollokation der elektromagnetischen mit der akustischen Welle nötig ist, keinen nennenswerten Einfluss auf das Schallfeld besitzt. 


\section{Danksagung}

Open access funding provided by Johannes Kepler University Linz. Die Autoren bedanken sich ausdrücklich für die finanzielle Unterstützung von der Österreichischen Forschungsförderungsgesellschaft (FFG) mit der Projektnummer 861570 und der voestalpine Stahl GmbH.

Open Access This article is distributed under the terms of the Creative Commons Attribution 4.0 International License (http://creativecommons.org/ licenses/by/4.0/), which permits unrestricted use, distribution, and reproduction in any medium, provided you give appropriate credit to the original author(s) and the source, provide a link to the Creative Commons license, and indicate if changes were made.

\section{Literatur}

1. Bernhard, F. (2014): Handbuch der Technischen Temperaturmessung. Berlin: Springer. ISBN 978-3-642-24506-0.

2. Schuster, S., Scheiblhofer, S., Reindl, L., Stelzer, A. (2006): Performance evaluation of algorithms for SAW-based temperature measurement. IEEE Trans. Ultrason. Ferroelectr. Freq. Control, 53, 1177-1185.

3. Exel, D., Zagar, B., Schuster, S., Ganglberger, V., Reisinger, J. (2017): Parameter estimation and performance bounds for radiometric model-based non-contact temperature measurement. In IEEE international istrumentation and measurement technology conference, Turin, Italien (S. 641-646).

4. Exel, D., Zagar, B., Schuster, S., Ganglberger, V., Reisinger, J. (2017): Unsicherheitsbetrachtungen der Temperaturmessung aus Daten einer Multispektralkamera. In Tagungsband der 5. Tagung Innovation Messtechnik (S. 92-98). Aachen: Shaker Verlag $\mathrm{GmbH}$

5. Kutia, J. R., Xu, W. (2014): A portable radio-acoustic sounding system for temperature profiling within the boundary layer, industrial electronics society. In IECON (S. 23082313).

6. Daas, B.M. (1996): Die elektromagnetisch-akustische Wechselwirkung und ihre Anwendung zur berührungslosen Entfernungs- und Temperaturmessung in geschlossenen Räumen. Aachen: Shaker Verlag. ISBN 3-8265-2148-X.

7. Bradley, S. (2008): Atmospheric acoustic remote sensing. Boca Raton: CRC Press. ISBN $0-8493-3588-4$

\section{Autoren}

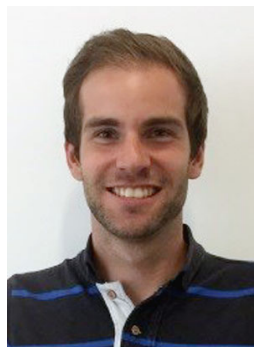

\section{Dominik Exel}

studierte Automatisierungstechnik an der Fachhochschule Wels, Österreich, und ist seit Mitte 2016 am Institut für Elektrische Messtechnik an der Johannes Kepler Universität in Linz, Österreich, beschäftigt. Im Rahmen seiner Dissertation beschäftigt er sich mit der berührungslosen Temperaturmessung. Seine Interessen liegen im Bereich der Signalverarbeitung und Sensorsysteme.

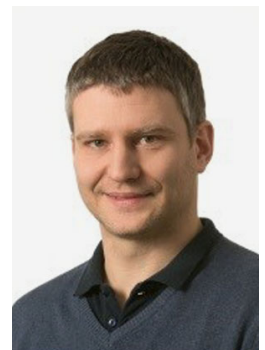

\section{Stefan Schuster}

wurde 1978 in Linz, Österreich, geboren. Er absolvierte das Mechatronik-Studium und die Dissertation an der Johannes Kepler Universität in Linz mit Schwerpunkt Messtechnik/Statistische Signalverarbeitung. Von 2007 bis 2009 war er als Senior Researcher am Christian Doppler Labor für Integrierte Radarsensoren am Institut für Nachrichtentechnik und Hochfrequenzsysteme an der Johannes Kepler Universität in Linz tätig. Seit 2009 ist er bei der voestalpine Stahl GmbH als Forschungsingenieur beschäftigt. Seine Interessen liegen im Bereich der statistischen Signalverarbeitung, Parameterschätzung, Radarsignalverarbeitung und RF-System-Design. Dr. Schuster erhielt den Österreichischen Mechatronikpreis 2007 und den IEEE Microwave Prize 2011
8. Daas, M., Knöchel, R. (1989): Compact sounding system using microwaves and ultrasound. In Microwave symposium digest, Long Beach, USA (S. 257-260).

9. Brooker, G., Martinez, J. (2008): Low-cost monostatic radio-acoustic sounding system for indoor temperature profiling. In Radar conference, Rome (S. 245-250).

10. Angevine, A. M. (2000): Radio acoustic sounding system (RASS) application and limitations. In Geoscience and remote sensing symposium, Honolulu, USA (S. 1180 1182)

11. Saffold, J. A., Williamson, F., Ahuja, K., Stein, L., Muller, M. (1999): Radar-acoustic interaction for IFF applications. In Radar conference, Waltham, USA (S. 198-202).

12. Daas, M., Knöchel, R. (1992): Microwave-acoustic measurement system for remote temperature profiling in closed envirenments. In Microwave conference, Helsinki, Finland (S. 1225-1230).

13. Weiß, M., Knöchel, R. (2001): A monostatic radio-acoustic sounding system used as an indoor remote temperature profiler. IEEE Trans. Instrum. Meas. 50(5), 1043-1047.

14. Weiß, M., Knöchel, R. (2001): Monostatic radio-acoustic sounding system. In Geoscience and remote sensing symposium, Hamburg, Germany (S. 29-31)

15. Richards, M. A. (2014): Fundamentals of radar signal processing. New York: McGrawHill. ISBN 978-0071798327

16. Marshall, J. M., Peterson, A. M., Barnes, A. A. (1972): Combined radar-acoustic sounding system. Appl. Opt., 11(1), 108-112.

17. Brooker, G., Johnson, D., Martinez, J., Robertson, D. (2015): A new modality radaracoustic sensor for visualisation in environments with poor radar/lidar reflectivity. In Workshop on alternative sensing for robot perception, IROS.

18. Thaller, K. (2018): Messaufbau zur interferometrischen Schallfeldmessung mit tomografischer Rekonstruktion, Linz, 2018.

19. Skolnik, M. (1981): Introduction to radar systems. New York: McGraw-Hill. ISBN 0-07-057909-1.

20. Harris, F. J. (1978): On the use of windows for harmonic analysis with the discrete Fourier transform. Proc. IEEE, 66, 51-83.

21. Kay, S. M. (1993): Fundamentals of statistical signal processing-estimation theory New York: Prentice-Hall.

22. Schuster, S., Scheiblhofer, S., Stelzer, A. (2006): DFT frequency estimation threshold level with windowed data. In International istrumentation and measurement technology conference, Sorrento, Italien.

23. Schuster, S., Scheiblhofer, S., Stelzer, A. (2009): The influence of windowing on bias and variance of DFT-based frequency and phase estimation. IEEE Trans. Instrum. Meas. 58(6), 1975-1990

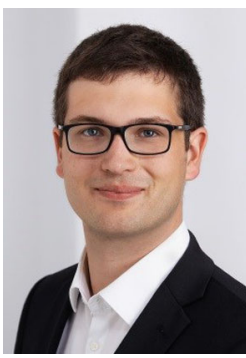

\section{Kurt Thaller}

schloss das Mechatronik-Studium 2018 an der Johannes Kepler Universität in Linz, Österreich, ab. Seit Mitte 2018 arbeitet er als HiL Testing Engineer bei Magna Powertrain.

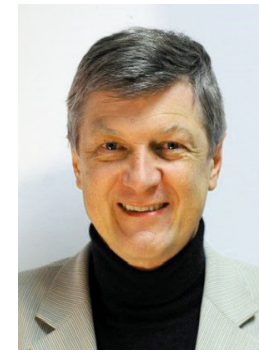

\section{Bernhard Zagar}

leitet das Institut für Elektrische Messtechnik an der Johannes Kepler Universität in Linz, Österreich. Seine Interessen sind im Fachgebiet Messtechnik weit gestreut und decken das Thema vom Sensordesign bis hin zur Signalverarbeitung ab. Besondere Schwerpunkte lagen bisher im Bereich der optischen Messtechnik, der Bildverarbeitung und der Magnettomographie. 


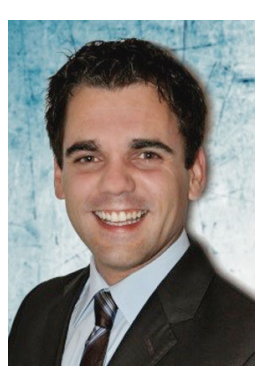

\section{Dominik Zankl}

wurde 1982 in Freistadt, Österreich, geboren. Er absolvierte das Mechatronik-Studium 2010 und die Dissertation 2017 an der Johannes Kepler Universität in Linz, Österreich, mit Schwerpunkt Messtechnik/Statistische Signalverarbeitung. Seit 2016 ist er bei der voestalpine Stahl GmbH als Forschungsingenieur beschäftigt. Seine Interessen liegen im Bereich der Radarsensorsysteme, Radarsignalverarbeitung, RF System Design und 3D-Topographie-Messungen.

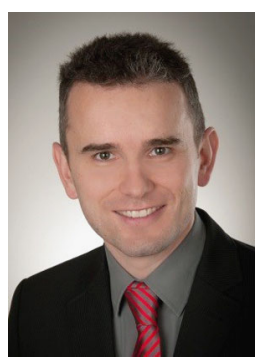

\section{Stefan Scheiblhofer}

wurde 1979 in Linz, Österreich, geboren. Nach dem Diplomstudium der Mechatronik an der Johannes Kepler Universität in Linz promovierte er 2007 zum Dr. techn. der Mechatronik auf dem Fachgebiet Radarsystemkonzepte und -simulation sowie Signalverarbeitung. 2007 bis 2010 setzte er seine Forschungstätigkeit am Christian Doppler Labor für integrierte Radarsensoren fort und wechselte danach als Systementwickler zu Hainzl Industriesysteme. Seit 2017 arbeitet er als Forschungsingenieur in der voestalpine Stah $\mathrm{GmbH}$ in Linz, mit Schwerpunkten auf den Gebieten der Radarsystemtechnik, magnetischen Messtechnik und Signalverarbeitung für Messsysteme.

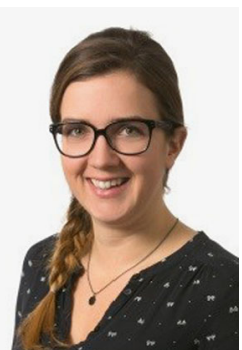

Vera Ganglberger

absolvierte das Studium der Mathematik und das Lehramtsstudium Mathematik/Englisch an der Universität Wien, Österreich. Seit 2011 arbeitet sie im Bereich der Algorithmen- und Sensorentwicklung und seit 2013 im Bereich Forschung und Entwicklung Mechatronik der voestalpine Stahl GmbH. Sie beschäftigt sich mit Signalverarbeitung und inversen Problemen aus dem Bereich der Temperaturmessung mit unterschiedlichen Messverfahren.

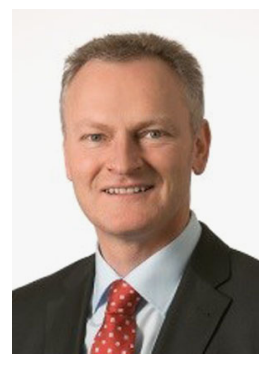

\section{Johann Reisinger}

ist Leiter des Bereichs Forschung und Entwicklung Mechatronik von voestalpine Stahl $\mathrm{GmbH}$, deren Schwerpunkte die Entwicklung von sensor- und modellbasierten Regelungssystemen sowie die Entwicklung von Simulatoren für die Prozess- und Materialentwicklung sind. Einen wesentlichen Aspekt bildet dabei die Anwendung dieser mechatronischen Systeme zur Optimierung von Prozessund Produktqualität.

Johann Reisinger ist Dipl.-Ing. der Technischen Physik und Doktor der Technischen Wissenschaften, er ist Autor von ca. 50 Publikationen mit den Schwerpunkten Sensorsysteme, Messtechnik, Modellierung, Regelung und Simulation in der Metallproduktion und verarbeitenden Industrie. 